\title{
EJEMPLOS DE MEMORIA, MNEMOTECNIA, PALLMPSESTO E HIPERTEXTO EN PANAMÁ Y EL MUNDO: UNA METODOLOGÍA PARA LA CONSERVACIÓN ${ }^{[*]}$
}

\author{
EXAMPLES OF MEMORY, MNEMONIC, PALIMPSEST AND HYPERTEXT IN PANAMA AND THE \\ WORLD: A METHODOLOGY FOR CONSERVATION
}

SILVIA ARROYO DUARTE ${ }^{* \star]}$

Fecha de recepción: 29 de marzo de 2017

Fecha de aprobación: 30 de abril de 2017

\section{RESUMEN}

En este artículo se analizarán los conceptos de memoria, mnemotecnia, palimpsesto e hipertexto. Sebastien Marot (2003), profesor de la École d'Architecture de la Ville \& des Territoires - Marne-la-Vallée (Francia), explica que estos cuatro reflejos son una nueva forma de afrontar un programa de diseño en arquitectura, el cual da prioridad al emplazamiento. Estos mismos conceptos -memoria, mnemotecnia, palimpsesto, hipertexto- están relacionados con la conservación, ya sea en un contexto urbano o arquitectónico. El objetivo de este trabajo es dar ejemplos (en su mayoría en Ciudad de Panamá) de su uso en esta disciplina, y vincular estos reflejos o características a un método para desarrollar un proyecto de conservación. Mediante estos ejemplos se explicará cómo la memoria y la mnemotecnia mantienen el espíritu del lugar, cómo un palimpsesto representa la historia de las transformaciones de un territorio, ciudad o edificio, y cómo ayuda un hipertexto a organizar un proyecto de conservación.

\section{PALABRAS CLAVE}

Memoria, mnemotecnia, palimpsesto

\section{ABSTRACT}

This article will analyze the concepts of memory, mnemonic, palimpsest and hypertext. Sebastien Marot (2003), professor of the École d'Architecture de la Ville \& des Territoires - Marne-la-Vallée (France), explains that these four reflexes are a new way of facing a design program in architecture, which prioritizes the site. These same concepts -memory, mnemonics, palimpsest and hypertext-are related to preservation, either in an urban or architectural context. The goal of this paper is to give examples (mostly in Panama City) of their use in this discipline, and relate these reflexes or characteristics to a method for the development of a conservation project. These examples will explain how memory and mnemonics preserve the spirit of place, how a palimpsest represents the history of the transformations of a territory, city or building, and how a hypertext can help organize a preservation project.

\section{KEYWORDS}

Memory, mnemonics, palimpsest

${ }^{*}$ )La idea de este artículo, que inició como un ensayo para la universidad, surgió al escuchar la conferencia titulada From the Art of Memory to the Art of Hope: A Little Odyssey (2012) y al leer el libro Sub-urbanismo y el Arte de la Memoria (2003), ambos del profesor Sebastien Marot (École d'Architecture de la Ville \& des Territoires Marne-la-Vallée).

(**) Doctora en Arquitectura, Edificación, Urbanística y paisaje por la Universitat Politècnica de València, España, con maestría Patrimonio Mundial por la Università degli studi di Torino, Italia, y en Restauración de Monumentos por la Universidad Politécnica de Catalunya, España. Licenciatura en Arquitectura Estructural por la Universidad Santa María la Antigua. Encargada del plan de manejo del sitio arqueológico de Panamá Viejo en el Patronato Panamá Viejo, y docente de la Facultad de Arquitectura de la Universidad de Panamá. Ha tomado cursos impartidos por la Agencia Española de Cooperación Internacional para el Desarrollo (AECID), el Swedish International Development Agency (SIDA) y el International Centre for the Study of Preservation and Restoration of Cultural Property (ICCROM). Contacto: viarroyo@hotmail.com 
Sebastien Marot (2012) define el sub-urbanismo como la poética del diseño, que se trabaja de lo general a lo particular. Es decir, desde el sitio al programa de diseño, del pasado al presente o de la ciudad a la arquitectura. Explica que los reflejos o características enraizadas en el diseño de jardines también caracterizan el enfoque del sub-urbanismo. Estos reflejos o características son la consideración por la memoria del sitio; la visión del sitio y el diseño como un proceso, no un producto; la lectura en profundidad de los espacios abiertos; y el sitio y el diseño como campos de conexiones y relaciones.

En su libro señala que esta teoría está inspirada en cuatro objetos: el libro El Arte de la Memoria de Frances Yates (publicado en 1966); el ensayo de Sigmund Freud "El Malestar en la Cultura", que contiene una comparación de la ciudad de Roma y la psique (publicado en 1929); el artículo de Robert Smithson sobre "Los Monumentos de Passaic" (1967); y el parque de Lancy en Suiza, diseñado por el arquitecto Georges Descombes (1979-1989). A continuación, Sebastien Marot explica las razones de tal inspiración:

- El libro de Yates analiza el uso de la mnemotecnia desde tiempos antiguos, a través de la época medieval, hasta que su uso finalmente migra a diferentes disciplinas, como la planificación urbana.

- Para explicar la complejidad de la psique, Freud la compara en su ensayo con la ciudad de Roma, que de cierta manera también ha sufrido "traumas" que se pueden haber borrado de la memoria (como un palimpsesto).

- Smithson describe en su artículo un paseo por la ciudad de Passaic, y concluye que al poner todas las ciudades del mundo una al lado de la otra, empezando con Roma y terminando con Passaic, cada ciudad se vuelve un espejo tridimensional que refleja la existencia de la siguiente.

- Finalmente, el parque de Lancy de Descombes es un instrumento de anamnesis o reminiscencia. En el diseño de este parque, el arquitecto construye en el palimpsesto, que constituye el territorio. Aborda sus diferentes capas al mismo tiempo, una al lado de la otra, conservando su legibilidad. Su trabajo es un mapa tridimensional del territorio, fragmentado y seccionado, que se vuelve legible o disponible en la memoria cuando uno lo visita.

La idea general de la conferencia del profesor Marot gira en torno a que los proyectos pueden activar la memoria de los sitios y las situaciones, sobre cómo leer las diferentes capas del paisaje y mantener la presencia de "algo que ya no existe pero que todavía está allí" (2012). Lo interesante es que Marot especifica como características del sub-urbanismo a la memoria del sitio, la visión del sitio como un proceso (palimpsesto), la lectura de espacios abiertos y los sitios como campos de relaciones (hipertexto). Todas estas palabras están también relacionadas con la conservación, ya sea en un contexto territorial, urbano o arquitectónico. El objetivo de este trabajo es dar ejemplos del uso de estas metáforas en la disciplina de la conservación, y vincular los reflejos o características del sub-urbanismo a una metodología que pueda emplearse para un proyecto de conservación.

A continuación, se definirán los conceptos de memoria, mnemotecnia, palimpsesto e hipertexto. Se mencionarán algunos ejemplos claros sobre cada concepto: uno en Afganistán (el caso del paisaje cultural y vestigios arqueológicos del Valle de Bamiyan), en España (la ciudad de Barcelona), en Italia (la iglesia de San Pietro Apostole y Santa María Assunta en Vilminore di Scave) y tres en Panamá (en el sitio arqueológico de Panamá Viejo y el distrito histórico de Panamá).

Como se observará a lo largo del artículo, estos conceptos no son nuevos, pero tanto los ejemplos panameños como la conclusión en cuanto al uso de la teoría de Marot como metodología para un proyecto de conservación representan una innovación. 


\section{Memoria}

La memoria siempre ha estado asociada a la arquitectura, el urbanismo y la conservación. Por ejemplo, Marot (2012) asevera que "la idea de la memoria en la arquitectura, la arquitectura como un instrumento de la memoria, la memoria como un material o como una dimensión de la arquitectura es un tema común".

Más de un siglo antes, John Ruskin explicaba que "debemos considerar muy seriamente la arquitectura como centralización y protección de esa sagrada influencia. Podemos vivir sin ella y adorar sin ella, pero no podemos recordar sin ella". De acuerdo con él, el tiempo es lo que hace a la arquitectura "histórica, conmemorativa o monumental" (1964, p. 203). Por lo tanto, se puede describir la arquitectura histórica o monumental como un monumento que representa la memoria de lo que debe ser conservado.

La relación entre monumento y memoria va mucho más allá, como indica Parrinello, que describe el monumento como "una señal para la memoria"1 (2011, p. 15), o la Carta de Cracovia, que define el monumento como "una entidad identificada por su valor y que forma un soporte de la memoria. En él, la memoria reconoce aspectos relevantes que guardan relación con actos y pensamientos humanos, asociados al curso de la historia y todavía accesibles a nosotros" (2000, anexo, punto 2).

Está claro que existe una conexión entre monumento y memoria a través de una línea de tiempo histórico que describe los procesos que han transformado un edificio y lo convierten en un monumento. Por ejemplo, Riegl lo llama "cadena evolutiva de la historia del arte" (1987, p. 25), Dezzi Bardeschi menciona un "proceso interrelacionado continuo" 2 (1990, p. 20) y Torsello explica que existe una "cadena ininterrumpida de pasados recientes que trazan una línea diacrónica muy especial" (2006, p. $116)^{3}$. Para conservar esta cadena histórica o línea del tiempo se debe mantener la autenticidad del monumento ${ }^{4}$.

Un ejemplo interesante sobre cómo la memoria ha jugado un papel clave en la conservación es el paisaje cultural y los vestigios arqueológicos del Valle de Bamiyan en Afganistán, inscrito en la Lista de Patrimonio Mundial y consecuentemente en la Lista de Patrimonio en Peligro en 2003. De acuerdo con la Organización de las Naciones Unidas para la Educación, la Ciencia y la Cultura (UNESCO),

...este sitio es un exponente de creaciones artísticas y religiosas características de la antigua Bactriana entre el siglo I y el XIII, en las que confluyeron distintas influencias culturales que desembocaron en la afirmación de la escuela de arte búdico del Gandhara. El sitio comprende varios conjuntos monásticos y santuarios budistas, así como edificios fortificados de la época islámica. El valle fue escenario de la trágica destrucción de las dos monumentales estatuas de Buda en pie, perpetrada por los talibanes en marzo de 2001, que causó una honda conmoción en el mundo entero. (2012)

\footnotetext{
1. Texto original en italiano: "La nozione di monumento riguarda un principio al contempo di tipo sensibile (la visione) e intelletuale (la memoria). II monumento è un segnale per la memoria, un monitore del ricordo, con un profondo significato morale e politico. II termine ha una precisa natura affettiva, giacchè non si tratta di trasmettere un'informazione neutra ma di scuotere, attraverso l'emozione, una memoria vivente" (Parrinello, 2011, p. 15).

2. Texto original en italiano: "Percepire la storia dell'architettura come continuo processo-intreccio, come sequenza dinamica di consistenze archeologiche in sedimentazione (e di usi collettivi in successiva archiviazione) è il modo più storicamente cosciente della propria partecipazione disciplinare" (Dezzi Bardeschi, 1990, p. 20).

3. Texto original en italiano: "Catena ininterrotta di passati prossimi che tracciano uno speciale percorso diacronico" (Torsello, 2006, p. 116).

4. Walter Benjamin explica que "la autenticidad de una cosa es la quintaesencia de todo lo que, en ella, a partir de su origen, puede ser transmitido como tradición, desde su permanencia material hasta su carácter de testimonio histórico" (2003, p. 22).
} 
La destrucción de estas estatuas ha estado en el ojo público por casi 15 años. Explica Marco Dezzi Bardeschi en la revista ANANKH que algunos meses después de lo sucedido una misión técnica encabezada por Vittorio Sgarbi fue a inspeccionar Bamiyan con la intención de reconstruir las estatuas. Al inspeccionar el sitio, cambiaron de idea: "imposible reconstruirlos, proclamó [Sgarbi]; es una locura reconstruirlos como un falso [histórico], como los japoneses, porque mientras menos los ves más lo sientes" (Dezzi Bardeschi, 2011, p. 2) ${ }^{5}$.

Entonces se desarrolló un proyecto para conservar el nicho en su estado actual, en la 9a reunión del grupo de expertos de Bamiyan, en marzo de 2011, con el apoyo de UNESCO. Señala el arquitecto Andrea Bruno (2011), quien formó parte del equipo, que los nichos vacíos representan un gesto de brutalidad que no puede ser perdonado, pero manera su vez continúan transmitiendo el mensaje que los talibanes querían eliminar, y la memoria todavía está allí. Cualquier reconstrucción sería un acto de violencia similar a la destrucción de los Budas, ya que eliminaría cualquier resto de autenticidad que le quedara al sitio. El proyecto presenta las esculturas en negativo, representadas por el alto relieve, dejando como protagonista a los nichos, tallados hace siglos por hombres guiados por su fe.

La historia no termina allí. En 2013, un grupo liderado por expertos alemanes del Consejo Internacional de Monumentos y Sitios (ICOMOS) decidió iniciar la reconstrucción de los pies de la escultura más pequeña sin la autorización de UNESCO. Dicha intervención incluía ladrillos, cemento y acero, y desde la perspectiva de algunos causó daños irreversibles. Este incidente pone de manifiesto la falta de consenso que existe entre la comunidad internacional sobre el tema de la reconstrucción (Bobin, 2015; Bevan, 2017).

A pesar de los desacuerdos, el proyecto apoyado por UNESCO fue enriquecido por el uso de la memoria como elemento clave para mantener la autenticidad y ayudar en la interpretación del sitio. La memoria de las estatuas se conserva en el espacio vacío de los nichos, que es mucho más expresivo y encamina a una interpretación mucho más clara que cualquier reconstrucción. La autenticidad también se preserva gracias a la línea de tiempo histórica que transforma a las estatuas en el monumento que son hoy en día, aunque parte de esta línea sea su destrucción. Como escribe Frances Yates, "Los dejo con la idea de que los edificios sean quizás menos sólidos de lo que aparentan, y que existen de forma invisible en la mente del arquitecto antes de materializarse; solo se recuerdan de manera invisible a través del tiempo en los recuerdos de las sucesivas generaciones" (1980, p. 23).

\section{Mnemotecnia}

La mnemotecnia es el arte que enseña memorizar a través de una técnica en la que se imprimen en la memoria lugares e imágenes (Yates, 2005). Es un proceso de asociación mental que facilita el recuerdo de algo. Como afirma Marot (2012), es una manera de recordar "algo que ya no existe pero que todavía está allí".

El fenómeno de la mnemotecnia puede observarse en diversos episodios de la historia. Por ejemplo, el ser humano busca para establecerse sitios geográficamente estratégicos con algún valor espiritual. Estos espacios -ya sea a nivel urbano o arquitectónico- se superponen o traslapan a través de la historia. Un caso típico son los procesos de la colonización o conquista. Por ejemplo, en lo que respecta a América y España en la época colonial, los españoles construyeron las iglesias católicas sobre los templos indígenas o las pirámides durante la ocupación del "Nuevo Mundo" para facilitar el recuerdo del lugar para los nuevos creyentes y también para mostrar poder sobre la

\footnotetext{
5. Texto original en italiano: "Impossibile rifarli; -proclamò-folle ricostruirli false, come vorrebbero i giaponesi: meno li vedi e più li senti" (Dezzi Bardeschi, 2011, p. 2).
} 


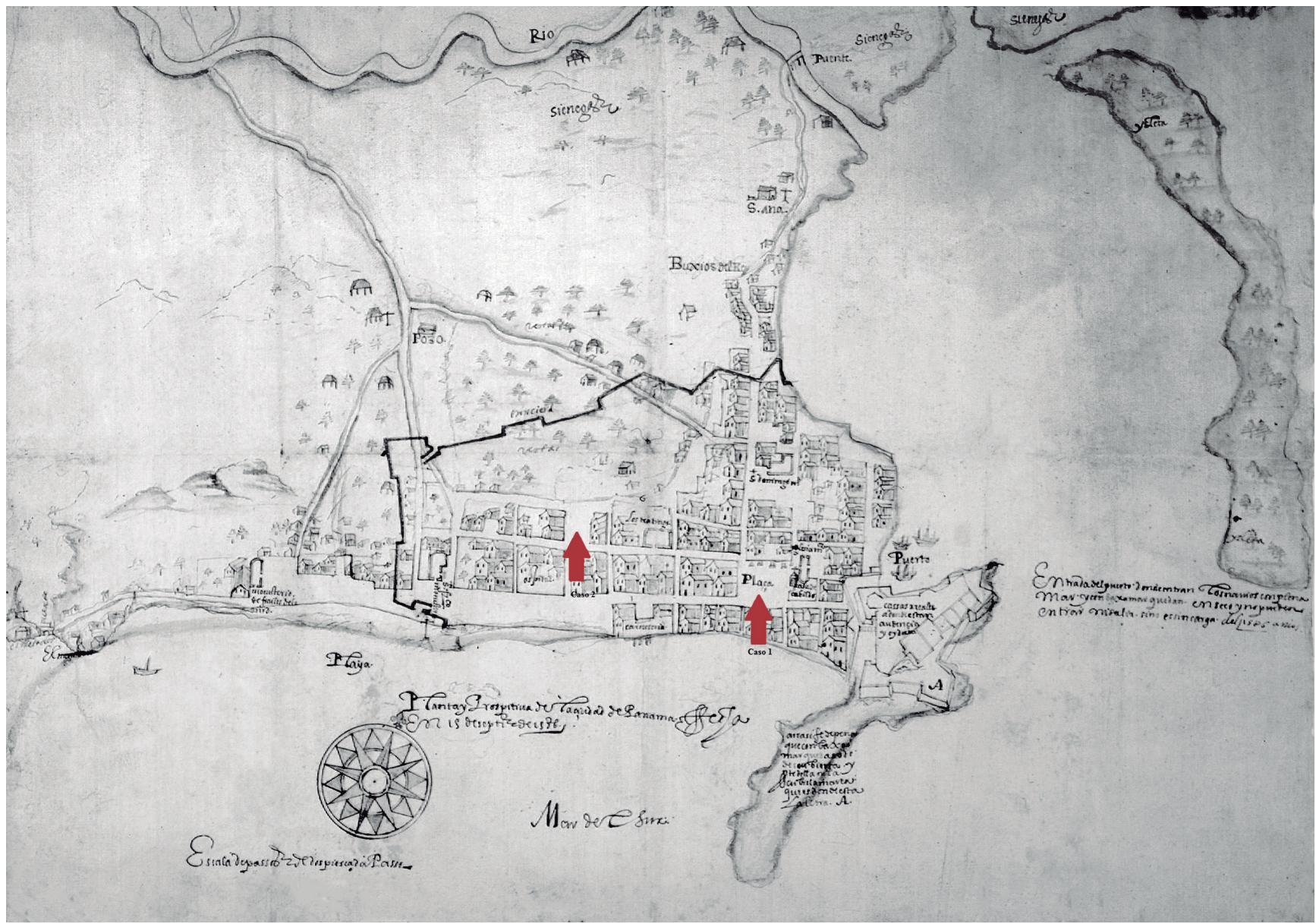

civilización conquistada. Esto también ocurre al revés, cuando el sitio es abandonado pero se mantiene su valor espiritual, como es el caso de los asentamientos de las tribus indígenas del noroeste de los Estados Unidos, que hasta hoy en día son visitados en ocasiones especiales para ceremonias rituales.

Se ha mencionado el uso de la memoria en proyectos de conservación, como el ejemplo en Afganistán. Ahora se mencionarán unos ejemplos de mnemotecnia en la propiedad sitio arqueológico de Panamá Viejo y distrito histórico de la ciudad de Panamá. Este es un sitio serial, son dos áreas diversas, con administraciones diferentes, incluidas en un solo sitio debido a su relación histórica. Ambas quedan bajo la protección del Instituto Nacional de Cultura de Panamá.

Panamá Viejo (en la República de Panamá) es la primera ciudad española en el Pacífico americano, fundada en 1519 sobre una aldea de pescadores. La antigua ciudad funcionó como centro de expedición y de rutas comerciales (ver Figura 1). Panamá Viejo prosperó e influenció el intercambio en la región hasta que en 1671 el pirata Henry Morgan atacó y saqueó la ciudad, que fue quemada, abandonada y utilizada como cantera para la construcción del nuevo asentamiento (Patronato Panamá Viejo, 2006).

El sitio arqueológico de Panamá Viejo estuvo abandonado por más de tres siglos y la moderna capital ha crecido a su alrededor, a lo largo del siglo XX, hasta la fecha. Actualmente combina las capas de desarrollo humano a través de diferentes etapas de la historia del continente. Los restos de sus períodos prehispánico y colonial, y una traza
Figura 1. Plano de la ciudad de Panamá, 1586

Fuente: Bautista Antonelli, Museo Naval de Madrid. 
devenir Vol. 4, N7, ENERO - JUNIO 2017, PP. 83-100 - ESTUDIOS I ISSN 2312-7562

UniverSidAd NACIONAL de INGENIERÍA, LIMA

Figura 2. Vista aérea del sitio arqueológico de Panamá Viejo, 2001

Fuente: Fotografía por Jean Christoph Henry (Patronato Panamá Viejo).

Figura 3. Vista aérea del sitio arqueológico de Panamá Viejo, 2014

Fuente: Fotografía por Félix Durán ( $\mathrm{Pa}$ tronato Panamá Viejo).

urbana única han sido preservados gracias al abandono por más de tres siglos de esta antigua ciudad (ver Figura 2). El sitio, que actualmente cuenta con 28 hectáreas, estuvo administrado por diferentes instituciones, hasta que en 1996 se creó el Patronato Panamá Viejo, institución sin fines de lucro que hasta la fecha lo gestiona y conserva (Patronato Panamá Viejo, 2006). En este sitio arqueológico relativamente pequeño se observan dos ejemplos de mnemotecnia que se mencionan a continuación (Figura 3).
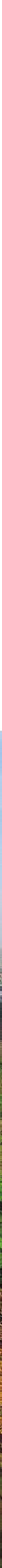


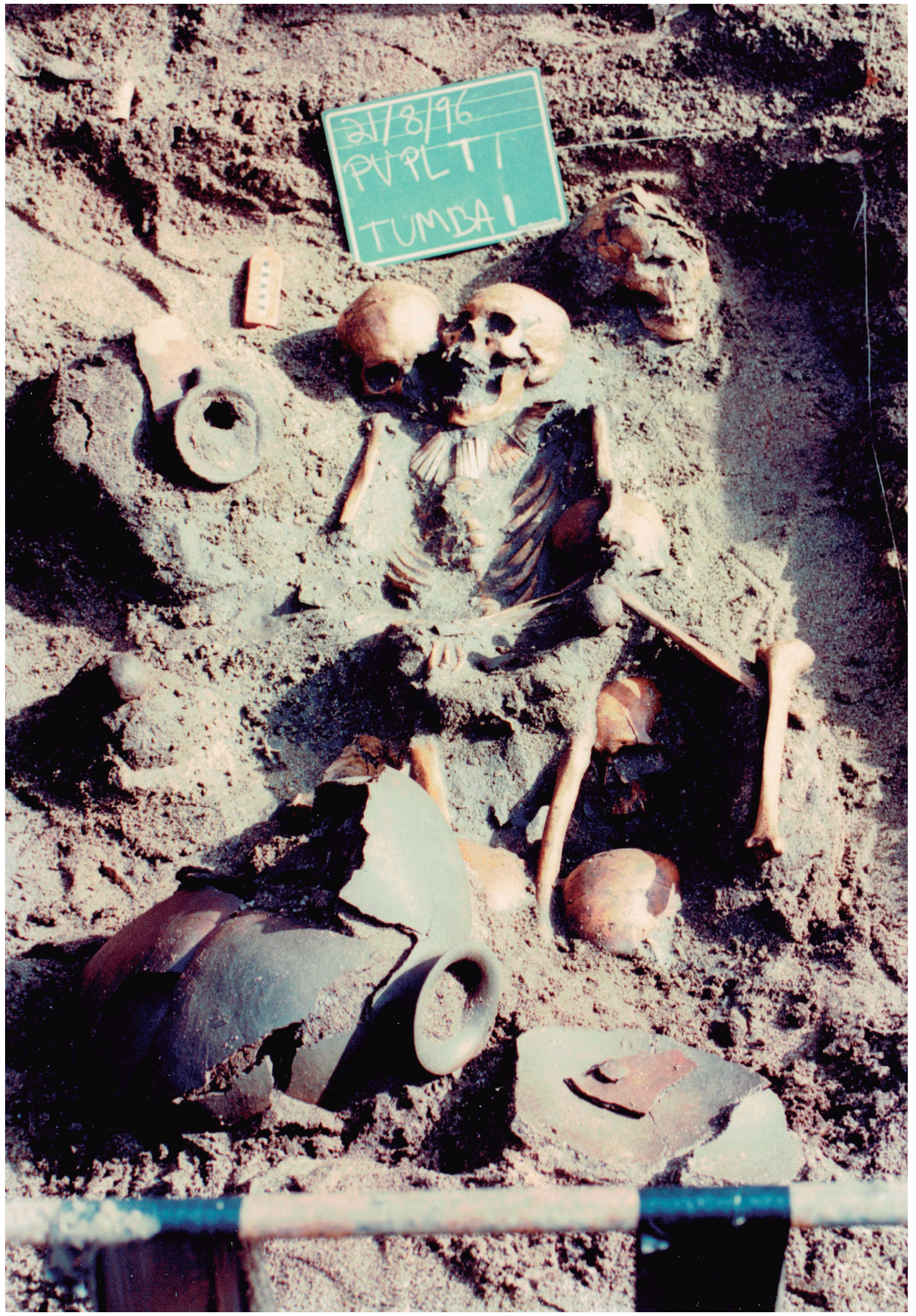

Figura 4. Vista de enterramiento prehispánico, actualmente en el museo del sitio arqueológico de Panamá Viejo, 1996 Fuente: Fotografía por Tomás Mendizábal, 1996. 


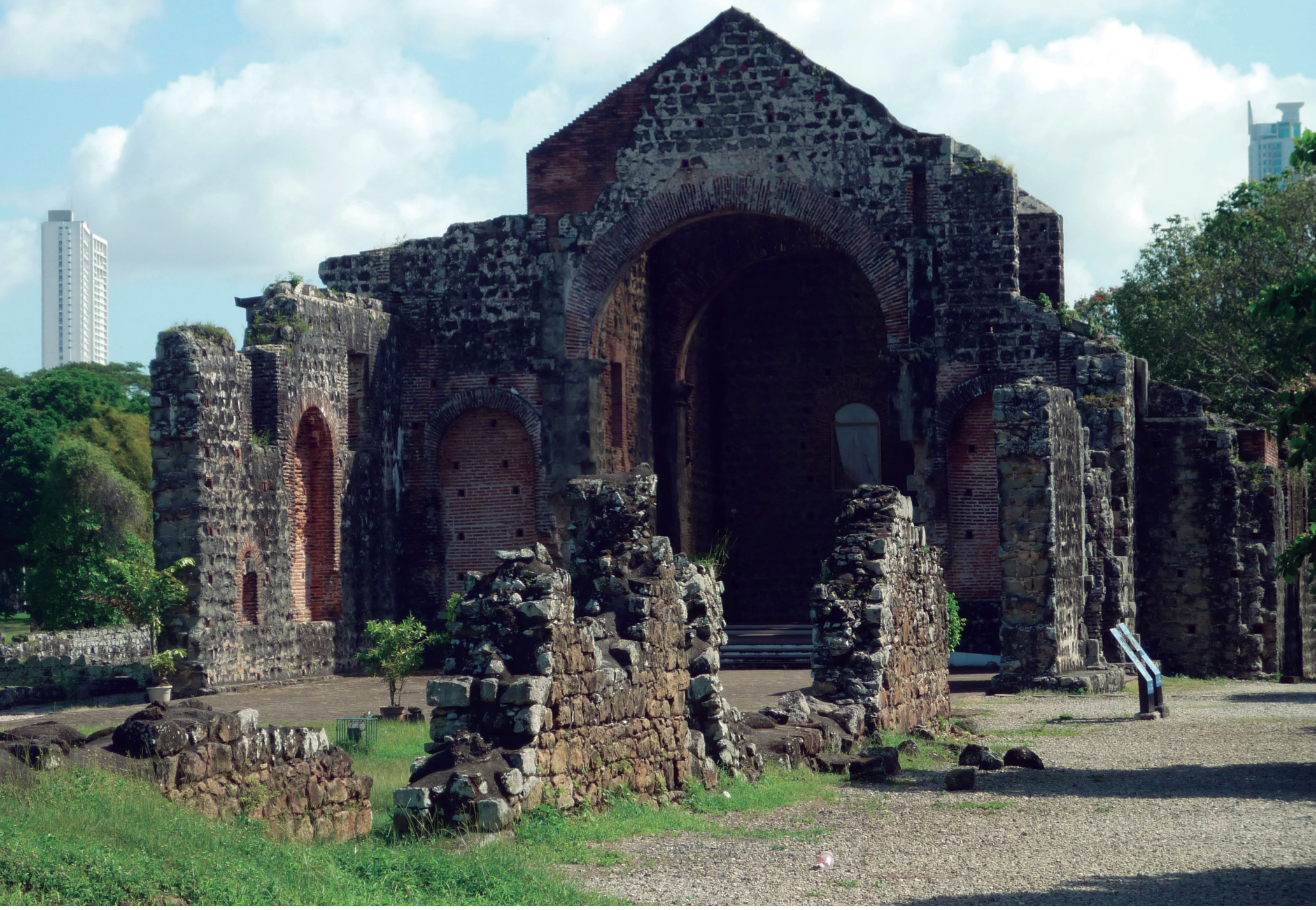

Figura 5. Convento de las monjas de la Concepción en el sitio arqueológico de Panamá Viejo, 2009

Fuente: Fotografía por Silvia Arroyo (Patronato Panamá Viejo).
En 1998 inicia el proyecto arqueológico Panamá Viejo, con la intención de excavar

...el área inmediatamente alrededor de la plaza Mayor del pueblo colonial, tratando de descubrir los restos arqueológicos y simultáneamente remover todos los elementos arquitectónicos modernos que distorsionaban la lectura del lugar". Lo interesante de estas excavaciones fue el hallazgo inesperado de "los restos milagrosamente conservados del asentamiento precolombino, justo debajo del centro del pueblo de los españoles. (Mendizábal, 2003, p. 41)

Se encontró tanto cerámica precolombina (utilizada por los pobladores de la aldea de pescadores) como varios enterramientos fechados entre el año 500 d. C. y el contacto con los europeos, que indican el posible uso ritual y funerario del sitio (Figura 4; Mendizábal, 2003).

Cabe mencionar que, al fundar la ciudad, los españoles encontraron el lugar perfecto para construir su plaza mayor justo sobre estos hallazgos prehispánicos de importancia ritual y funeraria. Este ejemplo de mnemotecnia definitivamente no fue casual: responde a la necesidad de fomentar el recuerdo del lugar entre los locales conquistados, así como de mostrar control y poderío sobre ellos apropiándose de sus espacios (posiblemente de culto).

En otra de las excavaciones del proyecto arqueológico Panamá Viejo, como parte de una escuela de verano de Arqueología en el convento de las monjas de la Concepción en 2003 , se encontró un enterramiento justo en el centro de las ruinas de la iglesia de 


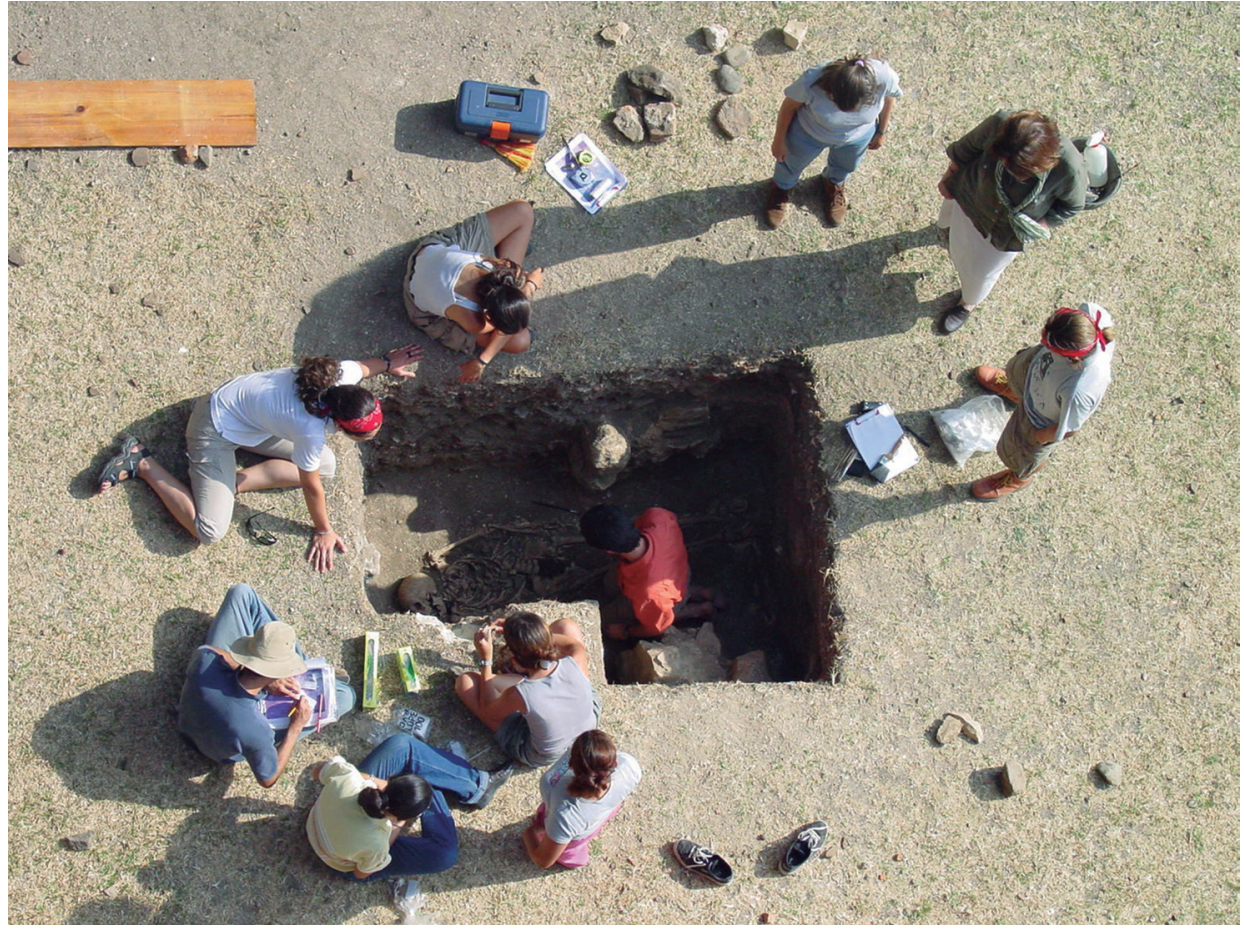

dicho convento, una de las más completas y representativas del sitio arqueológico (ver Figura 5). Estas ruinas mantienen la forma fácilmente identificable de un templo o iglesia. Al principio se pensó que el hallazgo era colonial. Al estudiarlo más de cerca, los arqueólogos, estudiantes y personal de campo se dieron cuenta de que se trataba un enterramiento de finales del siglo XIX o principios del siglo XX (ver Figura 6). Esta sepultura, realizada bajo las normas del ritual funerario cristiano, es consecuencia de que el sitio y, en este caso, esta iglesia, se mantienen en la mente de los hombres y mujeres a través del imaginario colectivo como un lugar sagrado, apropiado para rituales espirituales (Pereira, 2002).

En ambos casos, la mnemotecnia ayuda a mantener el recuerdo del espíritu del lugar. En el primer caso -la superposición de la Plaza Mayor sobre el asentamiento precolombino o prehispánico- el espíritu del lugar se mantiene porque, por un lado, se recuerda la importancia del lugar como espacio (posiblemente sagrado o de culto) para los locales. Por otro lado, recuerda la dominación de los españoles sobre los indígenas, incluso en su preciado territorio. En el segundo caso, es mucho más obvia la manera en que la imagen de la iglesia, incluso en ruinas, se conserva en la memoria como suelo sagrado, apropiado para rituales católicos como un funeral.

De acuerdo con Camila Mileto (2006), la mnemotecnia relaciona la historia, la memoria y el tiempo, que "son los ingredientes fundamentales del carácter de una arquitectura que se transforma, que muda y se estratifica, son los elementos que permiten que esta arquitectura se manifieste tal como es y que establezca con el hombre un diálogo cargado de mensajes" (p. 23).

\section{Palimpsesto}

La palabra palimpsesto se define como un "manuscrito antiguo que conserva huellas de una escritura anterior borrada artificialmente" (palimpsesto, s/f). Se utiliza mucho en contextos de arquitectura, urbanismo y conservación, al igual que la memoria y la
Figura 6. Vista de la Escuela de Verano de Arqueología trabajando en el enterramiento encontrado en el convento de las monjas de la Concepción, 2002

Fuente: Fotografía por Silvia Arroyo (Patronato Panamá Viejo). 


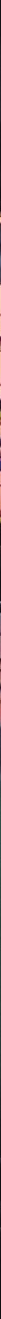

Figura 7. Vista del patio interior del Centro Excursionista en Barcelona, España, 2016

Fuente: Silvia Arroyo. mnemotecnia. Como ejemplo, se puede mencionar la interesante comparación que hace Corboz (1998) entre territorio y palimpsesto: "El territorio, Ileno de huellas y lecturas forzadas, se parece más bien a un palimpsesto" (p. 190).

Asimismo, Dezzi Bardeschi asocia la ciudad al palimpsesto:

Cada ciudad en que vivimos es un complejo, heterogéneo y estratificado palimpsesto, único material y estructuralmente, un recurso complejo e inseparable a mantenerse tal cual, minimizando las pérdidas en cultura material. En su permanencia encontramos nuestras raíces, nuestra cultura, nuestras referencias familiares, nuestras huellas, y la misma justificación de nuestros actos aquí y ahora 6 . (1990, p. 27)

Prácticamente toda ciudad con un pasado puede ser usada como ejemplo de la teoría del palimpsesto. Uno de los más bellos paradigmas de palimpsesto es la ciudad de Roma, explicado por Sigmund Freud: "todos esos relictos de la antigua Roma aparecen como unas afloraciones dispersas en la maraña de la gran ciudad de los últimos siglos a contar desde el Renacimiento, si bien es cierto que mucho de lo antiguo está enterrado todavía en su suelo o bajo sus modernos edificios". Freud continúa señalando que

6. Texto original en italiano: "Ogni città in cui viviamo è un complesso, eterogeneo palinsesto stratificato, un unicum strutturale e materico, una inscindibile risorsa complessiva da conservare come tale, minimizzando le perdite secche in cultura materiale. Nella sua permanenza ritroviamo le nostre stesse radici, la nostra cultura, i riferimenti familiari, i binari, la giustificazione stessa del nostro operare hic et nunc" (Dezzi Bardeschi, 1990, p. 27). 


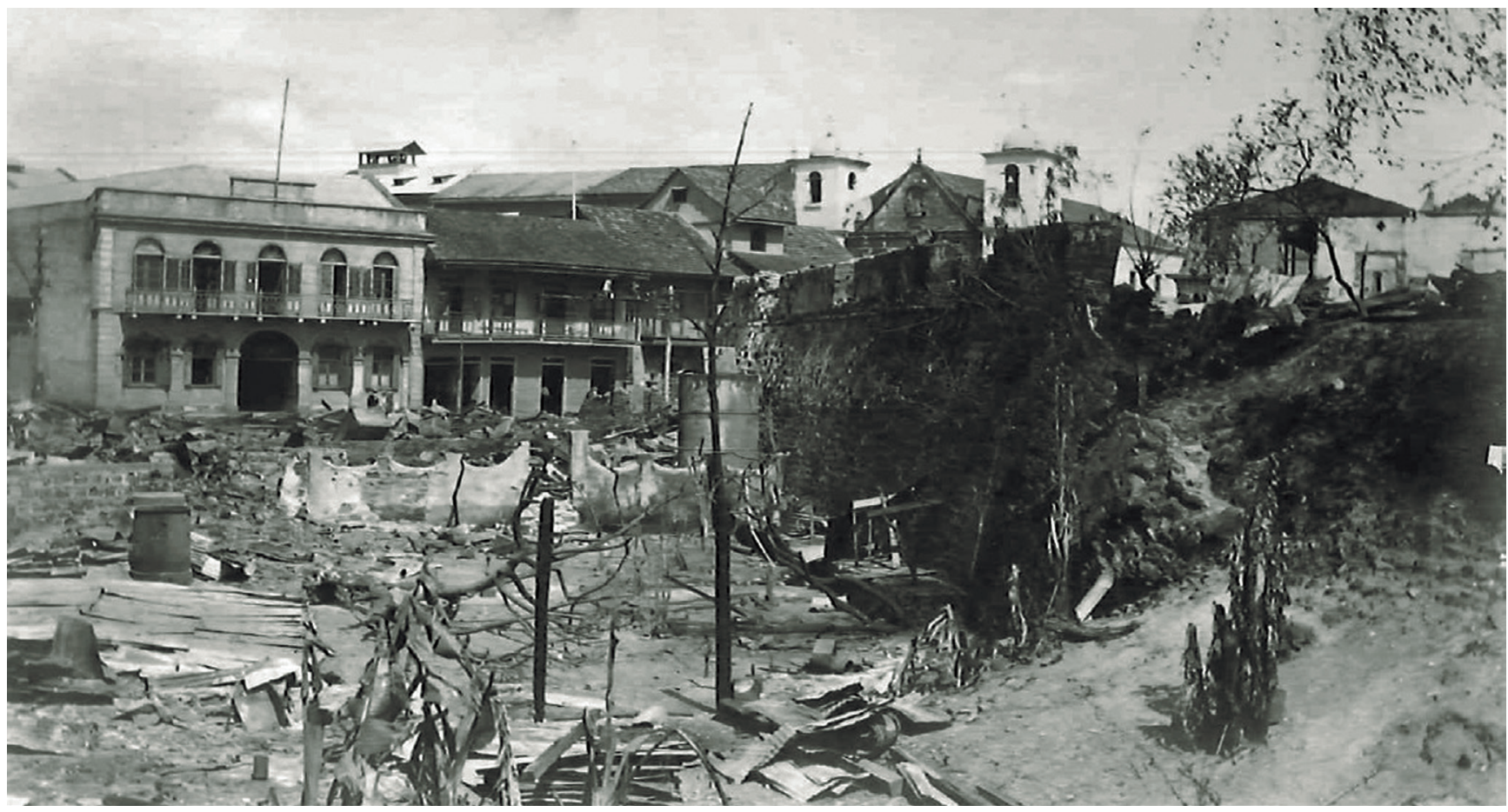

si “no se hubiera sepultado nada de lo que una vez se produjo, en que junto a la última fase evolutiva pervivieran todas las anteriores" (1929, pp. 70-71), símil que funciona perfectamente como definición de palimpsesto e igualmente para la conservación, a nivel urbano o arquitectónico, como uno de los criterios más importantes que debe tener un experto en esta disciplina. Asimismo, esta frase de Freud concuerda con el Artículo 11 de la Carta de Venecia:

Las valiosas aportaciones de todas las épocas en la edificación de un monumento deben ser respetadas, puesto que la unidad de estilo no es un fin a conseguir en una obra de restauración. Cuando un edificio presenta varios estilos superpuestos, la desaparición de un estadio subyacente no se justifica más que excepcionalmente y bajo la condición de que los elementos eliminados no tengan apenas interés, que el conjunto puesto al descubierto constituya un testimonio de alto valor histórico, arqueológico o estético, y que su estado de conservación se juzgue suficiente. El juicio sobre el valor de los elementos en cuestión y la decisión de las eliminaciones a efectuar no pueden depender únicamente del autor del proyecto. (1964, art. 11)

Otro ejemplo claro e interesante de una ciudad como palimpsesto es Barcelona, España. La Barcino romana, después de ser conquistada por los moros, continuó con su crecimiento durante la época medieval. En el siglo XVII fue construido su sistema defensivo, que duró alrededor de dos siglos y fue derribado en el siglo XIX para dar paso al plan de reforma y ensanche de lldefonso Cerdá (Morris, 1994). Todo esto puede percibirse al estudiar una fotografía aérea de la ciudad o simplemente caminando en sus barrios más antiguos. A través de un paseo por el Barrio Gótico de Barcelona uno puede encontrarse con el cardo o el decumanus -antiguas calles romanas que hoy en día son Carrer Llibreteria y Carrer del Bisbe-, y luego de unos pasos entrar en un edificio con rastros góticos, como el que hoy en día pertenece al Centro Excursionista, y encontrar los restos del templo romano dedicado a Augusto (Figura 7).

Un caso adicional de palimpsesto se presenta en el distrito histórico o Casco Antiguo de la ciudad de Panamá. Después de la destrucción de Panamá Viejo en 1671, la ciudad
Figura 8. Vista del actual Casco Antiguo o distrito histórico, donde se observa el derribo de la muralla después de un fuego $y$, al fondo, la Mansión Arias Feraud, 1906

Fuente: Biblioteca Roberto F. Chiari, Autoridad del Canal de Panamá 


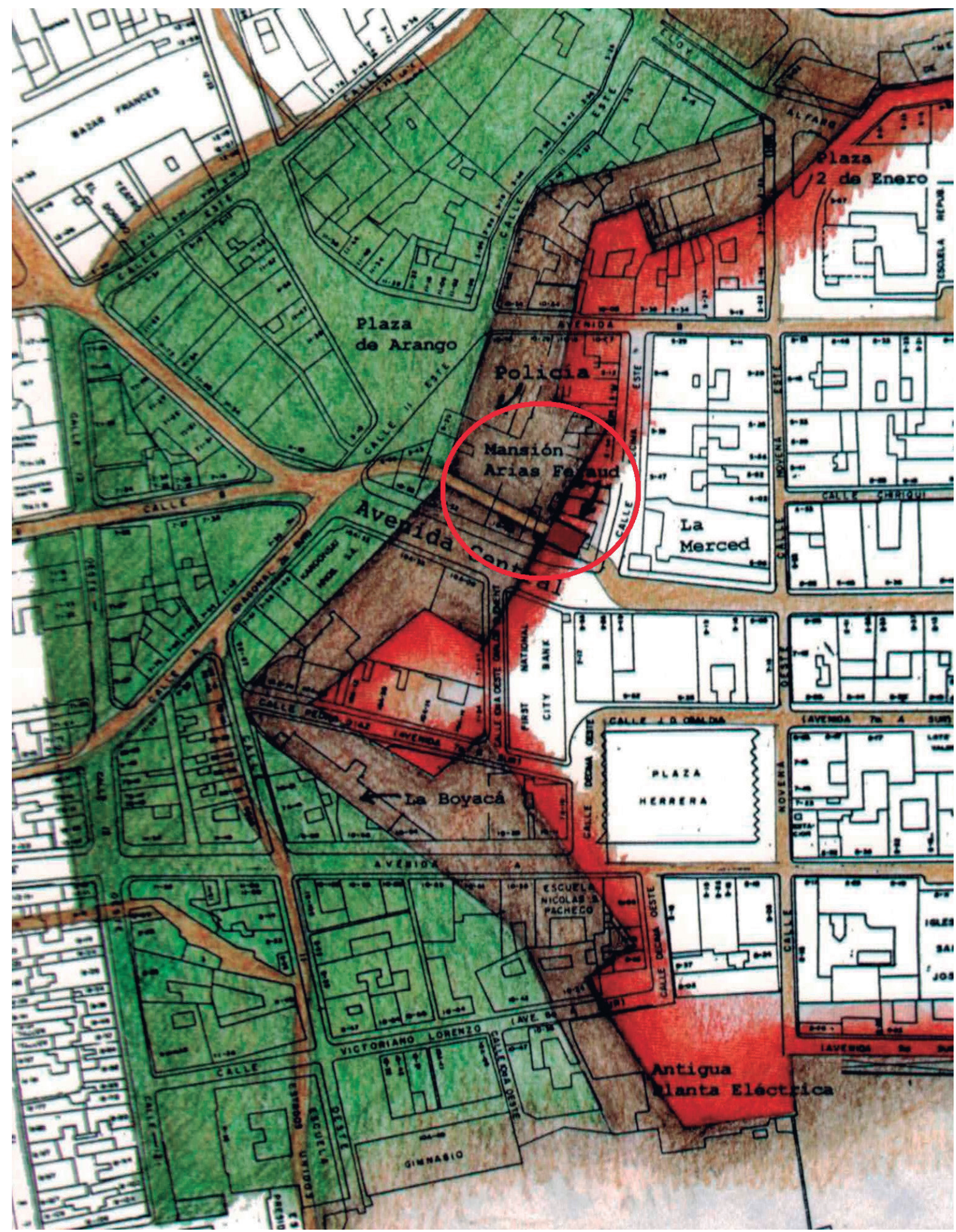

Figura 9. Plano actual del distrito histórico de Panamá sobre el cual se dibujó la fortificación, señalando los terrenos que esta ocupaba y el área de la Mansión Arias Feraud

Fuente: El Casco Antiguo de la ciudad de Panamá, por V. Spadafora y E. Tejeira Davis, 2001, Panamá, Panamá: Oficina del Casco Antiguo. 


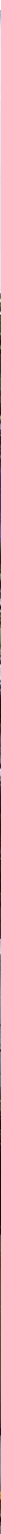

se trasladó al sitio de Ancón en 1673, lo que hoy en día se conoce como Casco Antiguo de la ciudad de Panamá (Patronato Panamá Viejo, 2006). Una de las razones por las que se escogió este lugar fue porque su ubicación resultaba beneficiosa para la defensa de la ciudad. Para no cometer los mismos errores del pasado, era necesario que "la urbe estuviese protegida de las acometidas de los piratas" (Carles, 1969, p. 205). Por consiguiente, un sistema defensivo se construyó entre 1673 y 1680 (Castillero Calvo, 2004).

Panamá continuó creciendo y las murallas cumpliendo su cometido hasta el siglo XVIII, cuando ya entorpecían su desarrollo. Como en muchas otras ciudades, se decidió que era necesario demolerlas. Esta tarea se llevó a cabo acatando la Ley del 11 de octubre de 1856, que dispuso la eliminación por el lado del continente de la muralla y su Puerta de Tierra (Carles, 1969, p. 208; Rovira, 1981, p. 34) (Figura 8).
Figura 10. Vista de la Mansión Arias Feraud o Casa de la Municipalidad, 2009

Fuente: Fotografía por Silvia Arroyo. 
Los terrenos sobre los cuales estaba construida la muralla pasaron a manos de particulares; en su mayoría fueron adquiridos por la Compañía del Ferrocarril para arrendarlos. Entre los que alquilaba un terreno a la compañía estaba Ramón Arias Feraud, quién allí erigió su casa, probablemente antes de 1881, fecha inscrita en la fachada (Rovira, 1981; Municipio de Panamá, 1981; Alcaldía de Panamá, 1993; Tejeira Davis, 2007) (Figura 9).

El Estado adquirió la mansión Arias Feraud oficialmente el 2 de septiembre de 1980. Para 1981 se decidió restaurarla, dándole así su actual función cultural y protocolar, hoy conocida como Casa de la Municipalidad. En su terreno, se realizaron excavaciones arqueológicas, gracias a las cuales se obtuvo gran cantidad de documentación y se encontraron los vestigios de la antigua Puerta de Tierra, parte de la muralla colonial y restos de la primera vivienda construida en el área (Rovira, 1981; Tejeira Davis, 2007). Estos hallazgos se pueden ver a través de una ventana arqueológica que hasta la fecha se mantiene en una de las habitaciones de la vivienda. Hoy en día, nuestro alcalde se mantiene "por azar o predestinación... sentado a las puertas de la ciudad" (Rovira, 1981, p. 36) (Figura 10).

Se puede observar a través de estos ejemplos que la cadena de procesos o acumulaciones llamada palimpsesto, como Dezzi Bardeschi (1990) explica, ayuda a formar una memoria colectiva y a tomar decisiones en la disciplina de la conservación, como qué mantener o cuándo innovar?

\section{Hipertexto}

De acuerdo con la Real Academia Española de la Lengua (RAE), hipertexto es un "conjunto estructurado de textos, gráficos, etc., unidos entre sí por enlaces y conexiones lógicas" (hipertexto, s.f.). El concepto es muy utilizado en las disciplinas de la información y la literatura. Es un texto compuesto de fragmentos de texto y los nexos que los conectan entre sí. Un ejemplo sencillo de un hipertexto es el de una nota al pie, que brinda información adicional al tema en cuestión (Landow, 1993).

La palabra hipertexto se aplica en el campo de la conservación con un significado análogo a la teoría del palimpsesto. Por un lado, como modelo de información para ayudar en la metodología de preservación; por otro lado, como una noción teórica, similar a la forma en que se utiliza en la literatura. A continuación, se presentan ejemplos de ambas teorías.

En 1998, Amedeo Bellini y Gian Paolo Treccani participaron en una reunión taller sobre Hipertexto y el Proyecto de Conservación en Vilminore di Scave (Bergamo, Italia). Las actas de esa reunión se publicaron un año después. El objetivo de este evento fue presentar un ejemplo del uso del hipertexto como metodología para un proyecto de conservación, utilizando como caso de estudio la iglesia de San Pietro Apostole y Santa María Assunta en Vilminore di Scave.

En las actas de esta reunión taller, Bellini (1998), preocupado por la manera en que las transformaciones se deben controlar en la disciplina de la conservación, explica sobre la "posible función del hipertexto para el desarrollo técnico del proyecto, que también se extiende a la gestión de la obra y la transmisión de comunicaciones entre el diseñador y el contratista" ${ }^{\prime \prime}$. En el mismo documento, Gian Paolo Treccani (1998) define hipertexto de la siguiente manera:

\footnotetext{
7. Texto original en italiano: "La sperienza-conoscenza del processo di accumulazione dei fatti urbani e territoriali produce memoria. L'orizzonte della memoria misura, in un dato momento, anche l'estensione delle previsioni dei comportamenti e delle scelte (cosa conservare, cosa innovare) è la memoria collettiva, che collega I possibili nodi di strutturarsi e di esprimersi dell'architettura" (Dezzi Bardeschi, 1990, pp. 19-20).

8. Texto original en italiano: "...possibile funzione dell'ipertesto per la formazione tecnica del progetto, che si estende anche alla gestione del cantiere, alla trasmissione delle comunicazioni tra progettista ed esecutore" (BeIlini, 1998).
} 
Un documento que consiste en un conjunto integrado de elementos de información (texto, gráficos, grabaciones, videos, etc.) que tiene funciones de consulta, no secuenciales sino guiadas por el flujo lógico de la búsqueda, y es un punto importante de progreso. En cualquier caso -como se ha podido observar-el sistema sirve de ayuda a la instrumentación tradicional, pero ciertamente no es un sustituto 9 .

Treccani (1999) continúa explicando el uso del hipertexto como metodología para un proyecto de conservación, utilizando como caso de estudio la iglesia de Vilminore di Scave. La aplicación de este sistema informático facilita el levantamiento sistemático de toda la documentación del proyecto: textos, imágenes, archivos, registros, dibujos, etc. Utilizando este sistema, el hipertexto puede ser útil para resolver cualquier problema que pueda producirse in situ, en el proyecto. Concluye afirmando que el objetivo de dicho sistema es desarrollar todo el potencial del hipertexto, incluyendo la interacción con otras bases de datos o motores de búsqueda. Con esto se obtiene un conocimiento más profundo del sitio y un juicio más preciso en los procesos de conservación.

Otro punto de vista es el de Concha Fernández Martorell (2004), que analiza el hipertexto desde el ángulo de la crítica literaria:

\footnotetext{
El fenómeno que la crítica literaria llama "hipertexto", escritura de una escritura, a modo de un palimpsesto que conserva el original (distintas versiones del mito de Don Juan, las meninas de las meninas), se revela de manera ejemplar en el ámbito de la arquitectura y la restauración; en ella el presente de la acción tiene que producirse necesariamente y de forma periódica, pues el monumento precisa de la restauración para su propia supervivencia y, en esa medida, la hace suya, la acción restauradora pasa a formar parte del monumento, en el conviven las distintas versiones con el original constituyendo un objeto único. Las condiciones que dieron nacimiento a la obra y los sentimientos históricos están presentes en el monumento. La manifestación simbólica inicial y sus significados posteriores, así como las formas de vida, costumbres, condiciones sociales, políticas, y económicas, los conocimientos, técnicas e ingenios, las de su origen y las que lo han mantenido en pie hasta nosotros, están testificados en el monumento; el contiene todo lo acontecido, es, por naturaleza, la representación viva de lo que la filosofía llama el Ser como ser histórico. (p. 15)
}

Sebastien Marot (2012) relaciona el hipertexto a la contemporánea ciencia de la información y el Internet. Para él, en un palimpsesto un texto se superpone al otro, y se puede descifrar o estudiar sus capas. En el hipertexto, los protocolos o conexiones ayudan a navegar entre los textos o documentos que no se relacionan entre ellos, y que no están catalogados u organizados.

El fenómeno del hipertexto busca desarrollar y administrar conexiones y relaciones a través de esta larga cadena de la transformación hasta llegar al monumento, sea a nivel territorial, urbano o arquitectónico. Representa un elemento clave, usado de forma teórica (como en el caso de la crítica literaria) o práctica (como en el campo de la información) para el mayor conocimiento del monumento a fin de mejorar la metodología y documentación de un proyecto de conservación.

\section{Conclusiones: Memoria, mnemotecnia, palimpsesto e hipertexto como parte de una metodología de conservación}

Los diferentes ejemplos que ilustran la relación existente entre la memoria, la mnemotecnia, el palimpsesto, el hipertexto y la disciplina de la conservación permiten comprender cómo la memoria y la mnemotecnia ayudan a mantener el espíritu del lugar;

9. Texto original en italiano: "Un documento costituito da un insieme di elementi informativi integrati (testo, grafica, registrazioni, video ecc.) che dispone di funzioni per una consultazione non sequenziale, bensi guidata dal flusso logico del criterio di ricerca, è un importante punto di avanzamento. In ogni caso - va affermato - si tratta di un sistema d'ausilio alla strumentazione tradizionale non certo sostitutivo" (Treccani, 1998). 
cómo un palimpsesto representa la historia de las transformaciones de un territorio, una ciudad o un edificio; y cómo se puede desarrollar un hipertexto para organizar un proyecto de preservación, creando un sistema de documentación que desarrolle un campo de conexiones y relaciones.

El monumento está compuesto por una larga cadena de procesos y transformaciones que se mantienen en las diferentes capas de la memoria, como un palimpsesto. Para conservar la memoria se utilizan técnicas como la mnemotecnia. Con esto, la permanencia y la autenticidad de un monumento se puede asegurar. Estas técnicas son herramientas que ayudan tener un conocimiento más profundo del sitio y un juicio más preciso en los procesos de conservación.

La teoría del palimpsesto ayuda a organizar estas capas o transformaciones -a nivel territorial, urbano o arquitectónico- de manera tridimensional y entendible. Si hay necesidad de llevar a cabo un proyecto de conservación, es importante desarrollar una metodología sistemática que registre todo el proceso, como lo permite el hipertexto.

La metodología de un proyecto de conservación puede compararse con los principios de sub-urbanismo que Sebastien Marot explicó en su libro y conferencia. El primer paso de esta metodología debe ser el levantamiento de la documentación o el conocimiento del sitio. Como en el primer principio del sub-urbanismo, el respeto por la memoria del sitio, se incluye el análisis de todos los procesos que han transformado el monumento a nivel territorial, urbano o arquitectónico.

Toda esta información ayuda a desarrollar una cronología o estratificación de la historia que, como un palimpsesto, hace más fácil visualizar el sitio como un conjunto de procesos, similar al segundo reflejo del sub-urbanismo. La última parte del método consiste en reunir toda esta documentación, leerla, y desarrollar conexiones y relaciones entre esta larga cadena de procesos, como en la teoría del hipertexto. Al igual que en el tercer y cuarto principio del sub-urbanismo, el objetivo es descubrir cómo el sitio se ha convertido en lo que es actualmente -esto es, su estado de conservación-.

Regresando al ejemplo de Panamá Viejo, el Plan Maestro de la Puesta en Valor del Conjunto Monumental Histórico ${ }^{10}$ de Panamá Viejo 1999-2009 menciona esta larga cadena de procesos y transformaciones como "la trayectoria histórica del sitio" que propone respetar"realizando solo las intervenciones mínimas necesarias para lograr este fin. No se proponen reconstrucciones puesto que la condición de ruina es una característica crucial e invariable de la autenticidad del sitio" (Patronato Panamá Viejo, 2006, p. 80; Law Environmental Caribe, 1999, p. 160).

En el plan de manejo 2014-2019 se han incluido algunas referencias hacia el paisaje, las capas históricas que el sitio posee y la lectura de la ciudad, claros referentes a la memoria, mnemotecnia y palimpsesto como parte inherente del sitio. Esto se observa de manera latente en uno de sus objetivos primordiales: "que Panamá Viejo se convierta en un centro de actividades científicas, educativas, interpretativas y culturales sin perder su imagen de ruina. Además, se debe conservar su entorno [paisaje] natural y cultural, sus capas pre hispánica, colonial y moderna, así como mejorar la lectura de la ciudad" (Arroyo, 2014, pp. 89-90).

De la misma manera, el plan de manejo incluye un sub-plan de investigación y actividades científicas, con el objetivo de "aumentar el conocimiento del sitio y divulgar la información", el cual busca, a corto plazo, desarrollar líneas de investigación para cada departamento técnico (Arqueología, Arquitectura y Conservación). A mediano plazo, busca crear 
...un centro de información para manejar toda la documentación del sitio arqueológico con la ayuda de un sistema de información, interno y externo, basado probablemente en sistemas de información geográfica (GIS, por sus siglas en inglés). Allí pueden almacenarse desde la información histórica, los informes, planos, fotografías, en fin, toda la documentación escrita y gráfica que se tenga del sitio. (Arroyo, 2014, pp. 126-127)

Este centro de información es equivalente al hipertexto que, como ya se mencionó, reúne la documentación, desarrolla y administra conexiones y relaciones entre esta larga cadena de procesos para descubrir las transformaciones del sitio.

\section{Referencias}

Arroyo, S. (2014). Plan de manejo del sitio arqueológico de Panamá Viejo [Documento inédito presentado al Patronato Panamá Viejo]. Panamá.

Bellini, A. (1998). Presentazione. En G. Cavagnini (Ed.). Ipertesto e progetto di conservazione. Vilminore di Scalve, Italia. Recuperado de http://www.scalve.it/giornatastudio/AmedeoBellini.htm

Benjamin, W. (2003). La obra de arte en la época de su reproductibilidad técnica. México: Ítaca. (Fecha original de publicación 1936)

Bevan, R. (enero, 2017). Ruin or rebuild? Conserving heritage in the age of terrorism. The Art Newspaper, 286. Recuperado de http://theartnewspaper.com/features/ruin-or-rebuild-conserving-heritage-in-an-age-of-terrorism/

Bobin, F. (enero, 2015). Dispute damage hopes of rebuilding Afghanistan's Bamiyan Buddhas. The Guardian. Recuperado de https://www.theguardian.com/world/2015/jan/10/rebuild-bamiyan-buddhas-taliban-afghanistan

Bruno, A. (2011). Il nuovo progetto per i Buddha. ANANKH, 63, 4-12.

Carles, R. D. (1969). 220 años del período colonial en Panamá. Panamá, Panamá: Talleres de Artes Gráficas de la Escuela de Artes y Oficios Melchor Lasso de la Vega.

Carta de Cracovia. Principios para la conservación y restauración del patrimonio construido. (2000). Recuperado de http://ipce.mcu.es/pdfs/2000_Carta_Cracovia.pdf

Carta de Venecia. Carta internacional sobre la conservación y restauración de monumentos y sitios. (1964). Recuperado de http://www.icomos.org/images/DOCUMENTS/Charters/venice_sp.pdf

Castillero Calvo, A. (2004). Historia general de Panamá (Vol. II). Colombia: Digital Designs Group.

Corboz, A. (1998). II territorio come palinsesto. En P. Viganò \& B. Secchi (Eds.), Ordine sparso: Saggi sull'arte, il metodo, la città e il territorio (pp. 177-191). Milán, Italia: Franco Angeli. (Fecha original de publicación 1983)

Dezzi Bardeschi, M. (1990). Restauro: punto e da capo. Milán, Italia: Franco Angeli.

Dezzi Bardeschi, M. (2011). Paesaggi con rovine: L'Aquila e Bamiyan. ANANKH, 63, pp. 2-3.

Fernández Martorell, Ch. (2004). Filosofía y restauración: El monumento como registro de la experiencia. Loggia, 16, 10-17.

Freud, S. (1992). El malestar en la cultura. En Sigmund Freud. Obras completas (Tomo XXI, pp. 65 140). Argentina: Amorrotu. (Fecha original de publicación 1929)

Hipertexto. (s.f.). En Diccionario de la Lengua Española. Recuperado en julio de 2012 de http://dle. rae.es/?id=KRjKpj5 [julio 2012]

Jackson, J. B. (1980). The necessity for ruins and other topics. Amherst, Massachusetts: University of Massachusetts Press.

Jackson, J. B. (1994). A sense of place, a sense of time. New Haven y Londres: Yale University Press.

Landow, G. P. (1995). Hipertexto: la convergencia de la teoría crítica contemporánea y la tecnología. Barcelona, España: Paidós.

Law Environmental Caribe (1999). Plan maestro de la puesta en valor del conjunto monumental de Panamá Viejo [Documento inédito presentado al Patronato Panamá Viejo]. Panamá. 
devenir Vol. 4, N7, ENERO- JUNIO 2017, PP. 83-100 - ESTUDIOS I ISSN 2312-7562

UNIVERSIDAD NACIONAL DE INGENIERÍ, LIMA

Marot, S. (1999). The reclaiming of sites. En J. Corner (Ed.), Recovering landscape: essays in contemporary landscape architecture (pp. 45-57). Nueva York: Princeton Architectural Press.

Marot, S. (2003). Sub-urbanism and the Art of Memory. Londres, Inglaterra: AA.

Marot, S. (6 de febrero, 2012). From the art of memory to the art of hope: A little odyssey [lecture]. Politecnico di Milano, Italia.

Mendizábal, T. (2003). Un siglo de arqueología en Panamá. Revista Cultural Lotería, (450/451), 34-49.

Mendizábal, T. (2005). Panamá Viejo: an analysis of the construction of archaeological time in eastern Panamá (Tesis doctoral sin publicar), University College of London, Reino Unido.

Mileto, C. (2006). La conservación de la arquitectura: materia y mensajes sensibles. Loggia, 19, 20-33.

Morris, A. E. G. (1994). History of urban form before the Industrial Revolution. Nueva York: John Wiley \& Sons.

Municipio de Panamá. (1993). Casa de la Municipalidad. Panamá: Servicios Malek. Recuperado de http://bdigital.binal.ac.pa/bdp/Casa\%20de\%20la\%20Municipalidad2.pdf

Organización de las Naciones Unidas para la Educación, la Ciencia y la Cultura. (2012). World Heritage Centre. Recuperado de whc.unesco.org

Palimpsesto. (s.f.). En Diccionario de la Lengua Española. Recuperado en julio de 2012 de http:// dle.rae.es/?id=RYgSAS [julio 2012]

Parrinello, R. (2011). Dal Monumentum al monumento: Percorsi lessicali e semantici. En S. Petrosin (Ed.), Monumentum: L'abitare, il politico, il sacro (pp. 13-22). Milán, Italia: Jaca Book Spa.

Patronato Panamá Viejo (2006). Panamá Viejo: De la aldea a la urbe. Panamá, Panamá: Autor.

Patronato Panamá Viejo (2013). Patronato Panamá Viejo. Recuperado de www.panamaviejo.org

Pereira, G. (2002). Análisis de un entierro encontrado en la iglesia del convento de las monjas de la Concepción de Panamá la Vieja. Revista digital Arqueología de Panamá la Vieja, 103-111.

Riegl, A. (1987). El culto moderno a los monumentos. Madrid, España: Visor. (Fecha original de publicación 1903)

Rovira, B. (1981). La arqueología en los proyectos de restauración: la mansión Arias Feraud en la ciudad de Panamá. Vínculos, Revista de Antropología del Museo Nacional de Costa Rica, 7(1-2), 33-36.

Ruskin, J. (1964). Las siete lámparas de la arquitectura. Madrid, España: Aguilar. (Fecha original de publicación 1849)

Spadafora, V. y Tejeira Davis, E. (2001). El Casco Antiguo de la ciudad de Panamá. Panamá, Panamá: Oficina del Casco Antiguo.

Tejeira Davis, E. (2007). Panamá: guía de arquitectura y paisaje. Sevilla, España: Consejería de Obras Públicas y Transportes.

Torsello, P. B. (2006). Figure di pietra: l'architettura e il restauro. Venecia, Italia: Marsilio.

Treccani, G. P. (1998). Ipertesto e progetto di conservazione. En G. Cavagnini (Ed.). Ipertesto e progetto di conservazione. Vilminore di Scalve, Italia. Recuperado de http://www.scalve.it/ giornatastudio/6_Treccani.html

Yates, F. (1980). Architecture and the art of memory. Architectural Association Quarterly, 10(4), 4-13.

Yates, F. (2005). El arte de la memoria. Madrid, España: Siruela. (Fecha original de publicación 1966) 